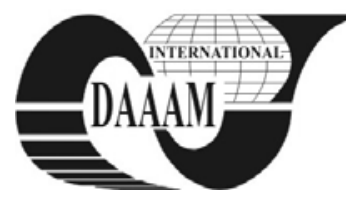

Annals of DAAAM for 2011 \& Proceedings of the 22nd International DAAAM Symposium, Volume 22, No. 1, ISSN 1726-9679 ISBN 978-3-901509-83-4, Editor B. Katalinic, Published by DAAAM International, Vienna, Austria, EU, 2011 Make Harmony between Technology and Nature, and Your Mind will Fly Free as a Bird Annals \& Proceedings of DAAAM International 2011

\title{
APPLICATION OF THE SIMULTANEOUS DSC/DTA/QMS THERMAL ANALYSE TO ALLOY AND NANOPOWDER CHARACTERIZATION
}

\author{
PESINA, Z[bynek] \& SOPOUSEK, J[iri]
}

\begin{abstract}
The unique simultaneous thermal analyse is introduced. The individualy adapted STA 409 / skimmer system deals with possibilites of application of thermal analysis methods. Methods of thermal analysis (DTA, DSC, QMS) were used to determine a wide range of thermodynamic quantities (e.g. melting point) of pure metal and alloys. DSC experiments were carried out for both bulk materials and for nanopowders. The paper also indicated the potential of CALPHAD method. Key words: DTA, DSC, QMS
\end{abstract}

\section{INTRODUCTION}

Thermal analysis is one of the most important methods of studying the composition and physic-chemical properties of materials. Due to dynamic development of this method there are many manufactures in the market (Setaram, TA Instruments, Netzsch and Linseis). Methods of thermal analysis are useful in studying different samples and monitoring their utility properties. These devices are usually equipped with options to operate different methods of thermal analysis and ordinarily associated with other analytical methods (thermogravimetry TG, mass spectrometry MS, interfered spectroscopy IR, Fourier transform infrared spectroscopy FTIR). The materials examined with the aid of thermal analysis can be very diverse.

Commercially available products are adapted to customer requirements. These devices are particulary suitable for use in basic research. This present contribution introduces one of these exceptional instruments. Its uniqueness lies in a connection of simultaneous thermal analysis with quadrupole mass spectrometer to STA 409 without skimmer system. This adaptation enables application of holder with Knudsen cell. The resulting configuration is capable of detecting extra-low gas pressures in metals and alloys.

\section{EXPERIMENTAL APPARATUS}

The simultaneous thermal analysis was carried out using individually adapted Netzch STA 409 skimmer apparatus for thermal analysis coupled with quadrupole mass spectrometer (QMS). The device enables to apply following instrumental methods: heat flow differential scanning calorimetry (DSC), differential thermal analysis (DTA) and Knudsen cell combined with mass spectrometry (KC/MS). The temperature range is (25-1400) ${ }^{\circ} \mathrm{C}$, rate of heating and cooling is within the range of (0.1-25) $\mathrm{K} / \mathrm{min}$. The gas (argon, nitrogen, air) flow range is $(0-300) \mathrm{ml} / \mathrm{min}$. The results measured can be treated via Protheus software. The different crucibles (Alumina, Pt) covered with protective layers can be used with respect to system under view.

\section{DSC}

The high sensitivity DSC was confirmed on various systems (e.g. Sn, Ag, Cu, Fe Ni, Al). The optimum of sample is approximately (10-30) mg. The high sensitivity of DSC measurement at the heating rate of $0.1 \mathrm{~K} / \mathrm{min}$ is demonstrated by example (lead free alloy) in Fig. 1 . Reduction of heat rate allows separating a complex signal, which is formed by overlapping large peaks and shoulders. The system is more close to equilibrium state and the transformation temperatures of the samples can be attained with approximately $0.3 \mathrm{~K}$ precision. Also, the high sensitivity enables also measurement of the heat effects of the transformations solid to liquid as well as in the solid state. The heat capacity measurement is also possible.

The better understanding of the experimental DSC signals can be achieved in connection to CALPHAD based method (Boettinger et al., 2006) compare with Fig.2. The CALPHAD approach enables each alloy studied to obtain the predicted temperature in dependence on the enthalpy function $(\Delta \mathrm{H})$ and the values of temperature enthalpy derivatives $\partial \Delta H / \partial T$ can consequently be used for comparison with DSC experimental signal.

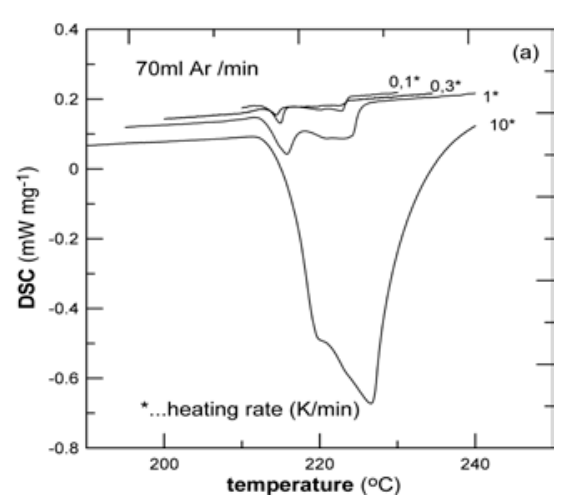

Fig. 1. Experimental DSC heating curves of sample Sn-1.0Ag0.5Cu-1.0Bi at different heating rates (Palcut et al., 2009)

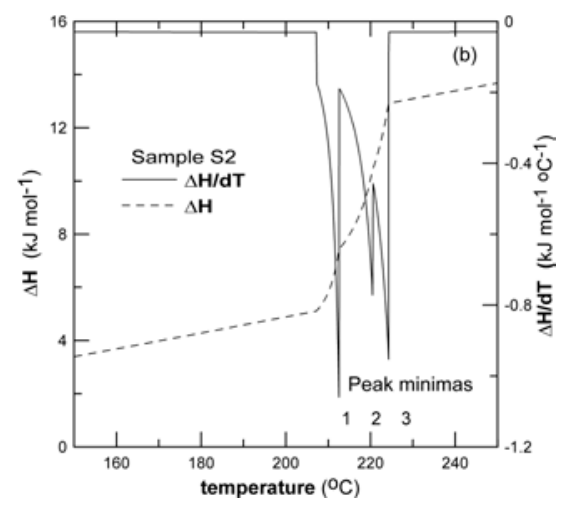

Fig. 2. Calculated molar enthalpies of the Sn-1.0Ag-0.5Cu$1.0 \mathrm{Bi}$ and the respective numerical temperature derivative (Sopousek et al., 2009) 
The DSC method can be used for bulk alloys as well as for nanopowders, see Fig. 3. The melting point depression, which is the best known property of nanoalloys can be easily detected.

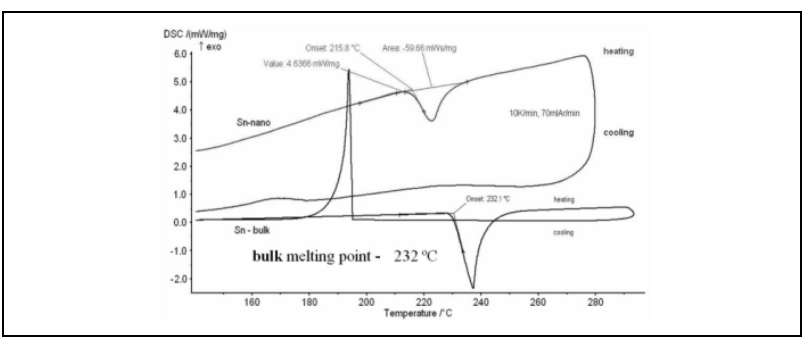

Fig. 3. The DSC signal for bulk and nanoscale tin (Broz et al., 2009)

\section{DTA}

After a simple holder change, the device Netzch STA 409 offers the possibility of running of the DTA method. For DTA samples both alumina crucibles and silica ampoules (temperature limit of silica being $1100{ }^{\circ} \mathrm{C}$ ) can be used. The method is suitable for determination of the phase transformations temperature of alloys. However, larger amounts of sample (approximately $0.5 \mathrm{~cm}^{3}$ ) than in the DSC method are needed. In sample sensitive to oxidation or high vapour pressure, using silica ampoules is unavoidable. Thus, the mass loss of sample is prevented and the observed signal is highly reproducible, see Fig. 4.

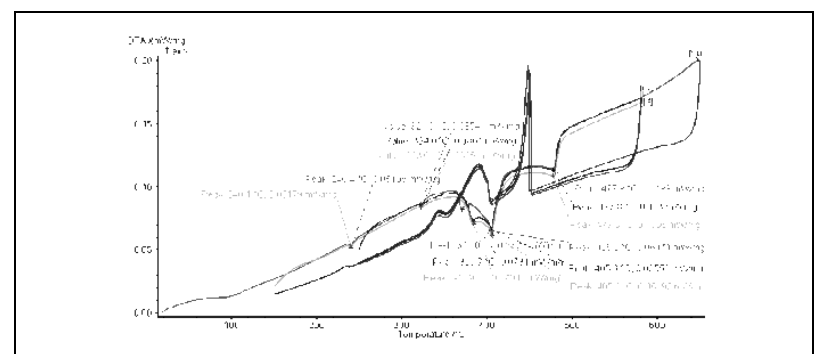

Fig. 4. The DTA signal of sample (Sb-23Sn-25Zn)

\section{KC/QMS}

The STA 409 / QMS (403/5) apparatus can be used for special measurement using Knudsen cell. This equipment consists of special single alumina crucible (Knudsen cell) covered by lid with small orifice. This crucible is placed on holder top with thermocouple. This arrangement can easily alternate with DSC or DTA holders. The volume (approx. 5 $\mathrm{cm}^{3}$ ) and diameter of Knudsen cell can be varied according to sample character. The diameter of the effusion orifice in the middle of the alumina lid is $1 \mathrm{~mm}$. The experiment using Knudsen cell starts with evacuation of the apparatus. The sample is heated under controlled temperature regime. The high temperatures can be reached in STA 409 (see Fig. 5) without the quadrupole mass spectrometer QMS function beeing affected.

The equilibrate sample gas phase is jetted from Knudsen cell into ionisation chamber of mass spectrometer. Ions are formed from gas by means of electron beam and separated inside QMS. Ion currents corresponding to gaseous species are detected. The activity of the metal elements forming alloys under view can be obtained by means of special thermodynamic procedure for both liquid and solid state measurements. The example of the activity determination is given in Fig. 6 (Sopousek et al., 2010)

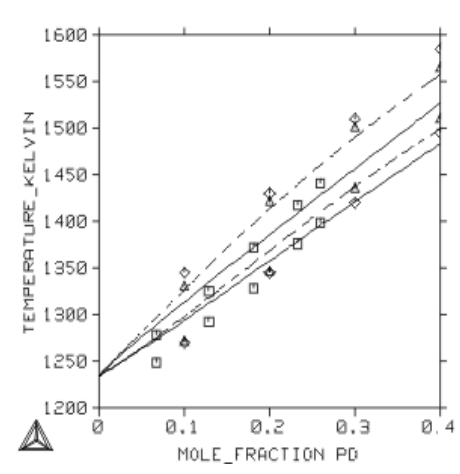

Fig. 5. Liquid and solidus temperatures calculated or predicted by different authors (lines) and selected experimental values (symbols)

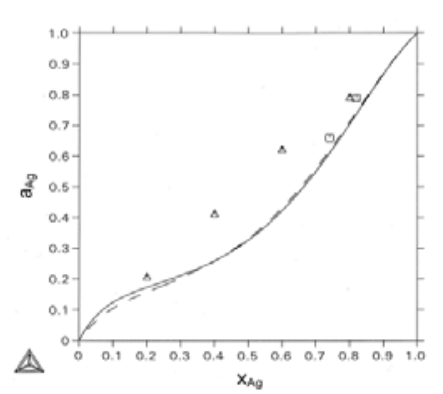

Fig. 6. Activity Ag in liquid AgPd system at 1873 K. Predicted dependences - lines, experimental values - symbols

\section{CONCLUSIONS}

DSC and DTA methods enable the temperature of phase transformations to be measured with high accuracy. DSC method is more suitable for heat effect evaluations. DSC and QMS measurement using Knudsen cell can be used for special applications as thermodynamic activity evaluations for metal element in alloys.

\section{ACKNOWLEDGEMENTS}

Financial support from the Czech Science Foundation (grant No. 106/09/H035) and Ministry of Education, Youth and Sports of the Czech Republic (MSM0021622410, CEITEC) is greatefully acknowledged.

\section{REFERENCES}

Boettinger, W.J. \& Kattner, U.R. \& Moon, K.W. \& Pererezko, J.H. (2006). DTA and DSC Measurement of Alloy Melting and Freezing. Washington, National Institute of Standards and Technology, 2010

Broz, P. \& Sopousek, J. \& Vrestal, J. (2009). On the stability of $\mathrm{Ag}, \mathrm{Cu}$ and $\mathrm{Sn}$ nanoparticles. In CALPHAD XXXVIII, Prague 17-22.5. 2009, pp. 118-118

Palcut, M. \& Sopousek, J. \& Trnkova, L. \& Szewczykova, B. \& Hodulkova, E. \& Ozvold, M. \& Turna, M. \& Janovec, J. (2009). Thermal analysis of selected tin-based lead-free solder alloys. Metallic materials 47/1, pp. 43-50

Sopousek, J. \& Palcut, M. (2009). The interpretation of the DSC signals of the lead-free solder alloys using the CALPHAD approach. In CALPHAD XXXVIII, Prague 1722.5. 2009, pp. 167-167

Sopousek, J. \& Zemanova, A. \& Vrestal, J. \& Broz, P. (2010). Experimental determination of phase equilibria and reassessment of Ag-Pd system. Journal of Alloys and Compouds. Vol.504., No.2, 2010, pp. 431-434 